\title{
TRADUÇÃO
}

\section{A esfera pública: ideologia e/ou ideal?*}

Amy Allen**

Lendo Mudança Estrutural da Esfera Pública (MEEP) de Habermas, cinquenta anos após sua publicação inicial, não se pode deixar de ficar impressionado com a cegueira da obra para as dimensões de gênero da esfera pública burguesa. Habermas não apenas ignora as formas em que esta esfera foi fundada sob a exclusão das mulheres, que estavam confinadas ao privado, ${ }^{1}$ ele o faz reconhecendo outros aspectos excludentes da esfera pública burguesa - sua exclusão de trabalhadores e camponeses - e, ao mesmo tempo, valorizando a família burguesa por proporcionar um espaço de intimidade aos indivíduos particulares que se reuniam em público, a fim de discutir assuntos de interesse comum². Assim, não é apenas que o jovem Habermas não leva em conta as maneiras pelas quais a subordinação das mulheres está implicada na formação da esfera pública burguesa, cuja promessa perdida ele espera recuperar, nem que seja completamente cego às suas dimensões ideológicas. Em vez disso, ele parece ser seletivamente cego à subordinação de gênero. Para as feministas que se engajaram com MEEP após sua tradução para o inglês - especialmente aquelas que eram veteranas das disputas entre marxistas e feministas na década de 1970 sobre se o feminismo era uma distração burguesa do real empreendimento da revolução socialista - essa cegueira seletiva deve ter tocado um ponto nevrálgico. Para ser justo com Habermas, nenhum desses debates havia acontecido ainda quando ele escreveu MEEP - nesse sentido, o livro é, como todos os livros, um produto de seu tempo - mas é inquestionável que a recepção de seu trabalho pela teoria feminista americana foi filtrada por suas lentes.

Uma vez que se decide ler MEEP através das lentes da teoria feminista, no entanto, a exclusão de fato das mulheres da esfera pública burguesa parece bastante

* Tradução de Allen, A. (2012). The public sphere: ideology and/or ideal?. Political Theory, 40, p. 822-829. DOI: https://doi.org/10.1177/0090591712457664. Tradução de Amanda Soares de Melo (doutoranda em filosofia pela Universidade Federal do ABC). E-mail: amandasdmelo@gmail. com. Autorização para tradução e publicação concedida pelo detentor dos direitos autorais: SAGE Publications, Inc. Copyright $\odot$ 2012. Os direitos do material republicado são propriedade de terceiros e a permissão para outra utilização posterior deve ser concedida pela SAGE.

** Penn State University, Pensilvânia, Estados Unidos.

1 Neste ponto, veja as contribuições de Joan Landes e Mary Ryan em Habermas and the Public Sphere, ed. Craig Calhoun (MIT Press, 1992).

2 Sobre a exclusão de trabalhadores e camponeses, ver Mudança Estrutural, p. 221-237 e 297-298; sobre o papel da família na formação da esfera pública burguesa, ver p.164-176. 
óbvia. Mas as críticas feministas da obra enfatizaram, a saber, que a exclusão das mulheres da esfera pública burguesa não é acidental ou contingente, mas antes constitutiva desse espaço. Se a esfera pública burguesa é constitutivamente excludente, então como Nancy Fraser coloca em sua crítica feminista extremamente influente de MEEP, "não podemos mais presumir que o modelo liberal da esfera pública burguesa era simplesmente um ideal utópico não realizado; era também uma noção ideológica" que serviu para racionalizar uma forma historicamente emergente de dominação de classe, raça e gênero. ${ }^{3}$

Em resposta a essa linha de crítica, Habermas não só reconhece que a esfera pública burguesa exclui mulheres, mas, talvez surpreendentemente, ele admite que essa exclusão é uma característica constitutiva, e não contingente, da esfera pública burguesa. Embora a exclusão das mulheres compartilhe algumas características com a exclusão dos trabalhadores e camponeses, "ao contrário da exclusão dos homens desprivilegiados", assume Habermas, "a exclusão das mulheres teve um significado estruturante". ${ }^{4} \mathrm{E}$ ainda, o argumento feminista para a natureza constitutivamente excludente da esfera pública burguesa, insiste Habermas, não "descarta os direitos de uma inclusão irrestrita e de igualdade que são uma parte integral da autocompreensão da esfera pública liberal, mas ao invés disso apela a eles" 5 . Em outras palavras, ao afirmar que a esfera pública burguesa é baseada na exclusão constitutiva das mulheres, as feministas implicitamente apelam para os ideais centrais dessa mesma esfera pública - inclusão e participação igualitária. Além disso, os discursos universalistas da esfera pública burguesa têm um potencial de autotransformação que permite que a própria esfera pública seja transformada por meio de seu contato com movimentos sociais como o movimento feminista6 ${ }^{6}$ A esfera pública burguesa pode ser ideológica, mas não é mera ideologia. ${ }^{7}$

Isso sugere que a resposta adequada à crítica feminista não é rejeitar o ideal da esfera pública como um todo, mas sim reformular a concepção liberal-burguesa que Habermas articula em MEEP e desenvolver uma concepção alternativa, pósburguesa $^{8}$. Fraser delineia quatro pressupostos específicos da esfera pública burguesa que precisam ser reformulados, a fim de tornar o conceito útil para a teoria crítica feminista. Primeiro, o modelo burguês assume incorretamente que é possível para as pessoas colocarem entre parênteses hierarquias de status existentes e participarem como se fossem pares em discussões públicas de assuntos de interesse comum;

\footnotetext{
3 Fraser, 1997, p. 76.

4 Habermas, 1992, p. 428.

5 Idem, p. 429.

6 Idem, ibidem.

7 Habermas já havia reconhecido isso em MEEP, embora sem notar os aspectos de gênero dessa ideologia; ver Mudança Estrutural, p. 237, 359 e 486.

8 Fraser, 1997a, p. 76.
} 
contra esse pressuposto e, em conformidade com algumas das próprias reflexões de Habermas no final de MEEP, Fraser insiste que a igualdade social é uma condição necessária para a democracia política. Em segundo lugar, em MEEP, Habermas tende a falar como se uma única e abrangente esfera pública universal fosse necessária para um bom funcionamento da democracia; ${ }^{10}$ em contraste, Fraser afirma que uma multiplicidade de esferas contrapúblicas é preferível, especialmente em sociedades que são divididas por hierarquias ${ }^{11}$. Em terceiro lugar, Fraser questiona o pressuposto de que o discurso público deve ser restrito à deliberação sobre assuntos de interesse comum; em vez disso, ela argumenta, as questões sobre o que conta como um assunto de interesse comum e quem é capaz de traçar e defender as fronteiras entre o público e o privado devem estar, elas mesmas, abertas ao debate em público, de forma que nunca possamos traçar essa linha de forma definitiva. ${ }^{12}$ Em quarto lugar, Fraser questiona o pressuposto de que uma esfera pública que funcione bem depende de uma separação nítida entre a sociedade civil e o Estado; ao contrário, precisamos fazer uma distinção entre públicos fracos e fortes - o primeiro sendo o local da formação da opinião e da vontade e o último sendo o lugar da tomada de decisão política - e teorizar sua inter-relação. ${ }^{13}$

Posteriormente, em seu monumental trabalho de teoria jurídica e política, Between Facts and Norms, ${ }^{14}$ Habermas reformula sua concepção da esfera pública, incorporando alguns - mas não todos - aspectos dessa concepção revisada, pósburguesa, da esfera pública. Especificamente, ele utiliza a noção de contrapúblicos subalternos de Fraser, com sua interpretação de públicos "alternativos” como lugares

9 Idem, p. 77-80. Pode-se argumentar que este é um dos principais pontos da argumentação de Habermas no encerramento da MEEP, no qual ele levanta dúvidas sobre se um discurso genuinamente inclusivo pode ser alcançado sem igualdade social - para os trabalhadores - $\mathrm{e}$ discute as perspectivas de uma esfera pública pós-burguesa. 0 ponto de Fraser, suponho, é que, em sua reconstrução histórica, Habermas não problematiza adequadamente esse pressuposto da concepção burguesa de esfera pública, particularmente no que se refere à exclusão das mulheres.

10 Habermas discute brevemente o papel das "esferas públicas intraorganizacionais" - o que ele entende por partidos políticos e grupos de interesses especiais - na criação de possibilidades para "publicidade crítica". Ver MEEP, p. 500-510. A essa altura, ele parece bastante pessimista quanto às perspectivas que essas esferas públicas têm de combater os efeitos niveladores de opiniões de massa gerados pela indústria cultural. Claro, essa situação teria parecido bastante diferente na época em que a crítica de Fraser foi escrita, dado o surgimento, entretanto, de novos movimentos sociais como o feminismo da segunda onda, que, ao invés de partidos políticos, servem de modelo para os contrapúblicos subalternos de Fraser.

11 Fraser, 1997a, p. 80-85.

12 Idem, p. 85-89. Para uma discussão relacionada, veja também "Sex, Lies, and the Public Sphere: Reflections on the Confirmation of Clarence Thomas," de Nancy Fraser em Justice Interruptus (1997) e "Models of Public Space: Hannah Arendt, the Liberal Tradition, and Jürgen Habermas" de Seyla Benhabib em Situating the Self: Gender, Community, and Postmodernism in Contemporary Ethics, 1992, p. 100.

13 Idem, p. 89-92.

14 Habermas, J. (1998). Between Facts and Norms: Contributions to a Discourse Theory of Law and Democracy. Tradução de William Rehg. Cambridge: MIT Press. 
de publicidade crítica, adotando explicitamente a distinção da autora entre públicos fortes e fracos e, ainda, fortalece sua interpretação de uma relação entre igualdade social e democracia política com sua análise de uma co-implicação entre autonomia pública e privada. ${ }^{15} \mathrm{Na}$ verdade, ele agora toma debates feministas sobre igualdade para fornecer o principal exemplo da necessidade de garantir conjuntamente a autonomia pública e privada. ${ }^{16}$ Como afirma: "nenhuma regulamentação [legal], por mais sensível que seja ao contexto, pode concretizar adequadamente o direito igual a uma vida privada autônoma, a menos que simultaneamente fortaleça a posição das mulheres na esfera pública política e, assim, aumente a participação nas formas de comunicação política que fornecem as únicas arenas em que os cidadãos podem esclarecer os aspectos relevantes que definem o estatuto de igualdade." 17 Portanto, os desenvolvimentos subsequentes de Habermas da teoria da esfera pública parecem corroborar a afirmação de que sua descrição da esfera pública pode ser reformulada para servir a fins feministas.

No entanto, desenvolvimentos históricos, políticos e teóricos subsequentes no intervalo de duas décadas levantaram novas questões sobre as exclusões constitutivas e distorções ideológicas em ação, mesmo nesta versão reformulada da teoria da esfera pública. Sob pressão para dar sentido às condições atuais da globalização, a teoria feminista, a teoria política e a teoria crítica passaram por uma virada global e essa virada mais uma vez lançou o conceito de esfera pública ao debate. Tal virada revela um conjunto diferente de exclusões na teoria da esfera pública de Habermas. Mesmo em sua versão reformulada, apresentada em Between Facts and Norms, esse modelo pressupõe implicitamente o que Fraser chama de "imaginário político Westfaliano", no sentido de que tacitamente assume que o quadro relevante para a esfera pública é o de "uma comunidade política limitada com seu próprio estado territorial". ${ }^{18}$ Justamente este quadro já não pode ser pressuposto à luz das condições presentes de publicidade transnacional. Em outras palavras, nas atuais condições de globalização, a esfera pública passou - e ainda está passando - por uma nova transformação estrutural. ${ }^{19}$

Em seu trabalho mais recente de teoria política, em particular, em suas reflexões teóricas e políticas sobre a União Europeia e as perspectivas de constitucionalização do direito internacional, Habermas refletiu sobre essa transformação. No que diz

15 Sobre públicos "alternativos", ver Between Facts and Norms, p. 373-374; sobre públicos informais (fracos) versus institucionais (fortes), ver p 306-308; sobre a relação entre igualdade e democracia, ver p. 420-423.

16 Ver Between Facts and Norms, p. 419-426 e "On the Internal Relation between the Rule of Law and Democracy," em The Inclusion of the Other: Studies in Political Theory de Habermas (Cambridge: MIT Press, 1998).

17 Habermas, 1998, p. 426.

18 Fraser, 2007, p. 8.

19 Idem, p. 15. 
respeito à Europa, baseando-se em seu argumento sobre o papel da esfera pública em garantir a legitimidade democrática em Between Facts and Norms, Habermas argumenta que o poder administrativo e político da União Europeia só pode ter legitimidade democrática na medida em que está enraizado em uma - ainda não existente - esfera pública europeia. ${ }^{20}$ Contra os eurocépticos, Habermas argumenta que a integração política europeia não precisa se basear numa identidade europeia pré-existente; em vez disso, as formas necessárias de identidade política europeia e solidariedade cívica poderiam ser geradas em uma esfera pública europeia transnacional devidamente configurada. ${ }^{21}$

No que diz respeito à esfera pública global, Habermas teme que as estruturas comunicativas emergentes das esferas públicas globais informais não possam ser eficazes enquanto não houver mecanismos constitucionalmente institucionalizados para traduzir a vontade pública gerada em tais esferas em poder político vinculante. Os protestos globais contra o início da guerra do Iraque em 2003 forneceram um exemplo comovente desse déficit de eficácia. Não obstante, Habermas é cautelosamente otimista de que as opiniões e vontades geradas em tais esferas públicas globais poderiam ser eficazes se dirigidas a uma instituição global - uma ONU dramaticamente reformada - que seria encarregada dos objetivos limitados de prevenir a violência e proteger os direitos humanos, dotada de força política para atingir esses objetivos. Para ser democraticamente legítima, essa instituição global teria de estar enraizada em uma esfera pública global, mas tal esfera não precisa ser mantida unida por formas grosseiras de identidade política ou solidariedade cívica; em vez disso, “a indignação moral compartilhada por violações flagrantes dos direitos humanos fornece uma base suficiente para a solidariedade entre os cidadãos do mundo" e a conquista de uma forma tão tênue de solidariedade global não é, na opinião de Habermas, um “obstáculo insuperável”. ${ }^{22}$

Certamente, alguém pode ser muito mais cético do que Habermas sobre as perspectivas atuais de desenvolver a solidariedade cívica europeia - especialmente à luz da atual crise financeira na Europa - ou um acordo global sobre o que constituem violações dos direitos humanos ou usos injustificados da violência nas relações internacionais. ${ }^{23}$ No entanto, mesmo deixando de lado esses temas difíceis, questões importantes podem ser levantadas sobre a legitimidade normativa desses

20 Habermas, 2006a, p. 102-106.

21 Habermas, 2006b, p. 80. Sobre esse ponto, veja também "The Postnational Constellation and the Future of Democracy," em The Postnational Constellation: Political Essays de Habermas (Cambridge: Polity Press, 2001), p. 98-103.

22 Idem, ibidem. Ver também "Does the Constitutionalization of International Law Still Have a Chance?" em The Divided West de Habermas (John Wiley \& Sons, 2004), p. 142-43, .

23 Sobre o último ponto, ver "Global Governance without Global Government? Habermas on Postnational Democracy" de William Scheuerman (Political Theory, 2008), p. 133-51. 
públicos transnacionais e globais emergentes. ${ }^{24}$ Considere que os participantes nas esferas públicas transnacionais e globais não são compatriotas que gozam de um igual status político, mesmo em um sentido formal, e que a estrutura das esferas públicas transnacionais e globais favorece as elites que possuem os recursos materiais e simbólicos necessários para a comunicação global, ${ }^{25}$ que possuem as habilidades linguísticas necessárias, incluindo a capacidade de se comunicar em inglês. ${ }^{26}$ À luz das enormes desigualdades globais, temos que nos perguntar se as opiniões e vontades debatidas e geradas em esferas públicas globais transnacionais e emergentes poderiam ser legítimas, no sentido de incluir todas as pessoas afetadas e permitir uma verdadeira paridade de participação. ${ }^{27}$ Pode-se até perguntar se a própria teoria da esfera pública está muito ligada ao contexto do lluminismo europeu - e, portanto, muito emaranhada com os legados do colonialismo e do imperialismo - para estar totalmente atenta a essas dinâmicas de poder.

Esta última questão surgiu de uma forma interessante em resposta à reconstrução transnacional pós-Westfaliano de Fraser da noção habermasiana da esfera pública. ${ }^{28}$ Os críticos de Fraser assinalam até que ponto sua estratégia de fundamentar a normatividade de sua concepção da esfera pública historicamente e não em uma teoria ideal - torna sua descrição particularmente vulnerável a tais preocupações. Dada esta sua estratégia normativa hegeliana, que Fraser compartilha com Habermas, a versão particular de história narrada por alguém tem grande importância. Como Hutchings coloca este ponto a respeito de Fraser (embora seja igualmente, se não mais, verdadeiro para Habermas), esta "é uma visão orientada para o futuro, informada por uma série de pressupostos sobre de onde viemos e para onde vamos (e, portanto, quem está na frente e quem está atrás). Esta é uma história que reflete o imaginário político específico que está intimamente ligado à experiência da modernidade ocidental em geral e ao destino dos Estados de bemestar liberal-capitalistas na última parte do século 20 em particular." ${ }^{29}$ Em outras palavras, não é suficiente para a teoria da esfera pública ser reformulada em um quadro mais amplo, transnacional, pós-Westfaliano, por mais importante e urgente

24 Fraser distingue críticas de eficácia e de legitimidade da esfera pública; estou mais preocupada aqui com a última. Ver "Transnationalizing the Public Sphere," e "Öffentlichkeit," em HabermasHandbuch, ed. Hauke Brunkhorst, Regina Kreide e Cristina Lafont (Stuttgart: JB Metzler Verlag, 2009).

25 Fraser, 2014, p. 16.

26 Idem, p. 18.

27 Idem, p. 19.

28 Veja as seguintes respostas ao ensaio de Fraser na mesma edição de Theory, Culture and Society: "Whose History? Whose Justice?" de Kimberly Hutchings, p. 59-63; "De-politicization of Democracy and Judicialization of Politics" de Shalini Randeria, p. 38-44; "The Exit from a Westphalian Framing of Political Space and the Emergence of a Transnational Islamic Public" de Armando Salvatore, p. 45-52; e "Transnationalizing the Public Sphere: A Critique of Fraser" de Oscar Ugarteche, p. 65-69.

29 Hutchings, 2007, p. 62. 
que este projeto seja; também precisamos interrogar as pressupostos implícitos e distorções ideológicas - sobre história, modernidade, progresso, esclarecimento e, finalmente, sobre a relação entre os Estados Unidos, a Europa e o resto do mundo que informam seus fundamentos normativos. ${ }^{30}$

No entanto, mesmo se assumirmos, com os críticos de Fraser, que a concepção pós-burguesa e pós-Westfaliana da esfera pública transnacional e global é ideológica, isso ainda nos deixa com uma versão da questão que Habermas colocou pela primeira vez há cinquenta anos em MEEP: é meramente ideologia? Em outras palavras, o conceito de esfera pública pode ser reconstruído de uma forma que o torne útil para uma teoria crítica que adotou não apenas uma perspectiva transnacional e pósWestfaliana, mas também pós-colonial? Não posso responder a esta pergunta aqui. Mas, para encerrar, gostaria apenas de observar que responder a esta questão exigirá interrogar criticamente a teoria modernista da história que sustenta a normatividade da teoria crítica habermasiana. ${ }^{31}$ Ao confrontar esta questão, também teremos que ser cuidadosos para não pressupor que sabemos como a inclusão e a igualdade no contexto das relações de poder neocolonial e neoimperial são possíveis, que sabemos que forma ela assumirá e quais regras ou procedimentos irão estruturá-la, pois fazer isso ameaça obscurecer, em vez de esclarecer, a natureza ideológica e as funções da nossa atual concepção de esfera pública.

\section{Referências}

Benhabib, S. (1992). "Models of Public Space: Hannah Arendt, the Liberal Tradition, and Jürgen Habermas" em Situating the Self: Gender, Community, and Postmodernism in Contemporary Ethics, New York: Routledge, p. 100.

Brunkhorst, H., Kreide, R., \& Lafont, C. (2009). “Öffentlichkeit” em HabermasHandbuch. Stuttgart: JB Metzler Verlag.

Calhoun, C. (1992). Habermas and the Public Sphere. Cambridge: MIT Press.

Fraser, N. (1997a) "Rethinking the Public Sphere: A Contribution to the Critique of Actually Existing Democracy" em Justice Interruptus: Critical Reflections on the "Postsocialist" Condition. New York: Routledge.

Fraser, N. (1997b). "Sex, Lies, and the Public Sphere: Reflections on the Confirmation of Clarence Thomas," de Nancy Fraser em Justice Interruptus: Critical Reflections on the "Postsocialist" Condition, New York: Routledge.

Fraser, N. (2007). "Transnationalizing the Public Sphere: On the Legitimacy and Efficacy of Public Opinion in a Post-Westphalian World" em Theory, Culture, and Society, no. 4, p. 7-30. DOI: https://doi.org/10.1177/0263276407080090.

30 Para uma importante discussão da relação entre o projeto normativo da teoria crítica habermasiana, com sua ênfase nas ideias de progresso e desenvolvimento humano e os legados do colonialismo, imperialismo e racismo, ver Race, Empire, and the Idea of Human Development de Thomas McCarthy (Cambridge: Cambridge University Press, 2009).

31 Sobre este ponto, ver também “Whose History?” de Hutchings (2007). 
Habermas, J. (1989). The Structural Transformation of the Public Sphere. Tradução de T. Burger. Cambridge: MIT Press. [Mudança estrutural da esfera pública: investigações sobre uma categoria da sociedade burguesa. Tradução de Denilson Luís. 1 a Edição. Editora Unesp, 2014].

Habermas, J. (1992). "Further reflections on the public sphere" em Habermas and the public sphere de Craig Calhoun, p. 421-461. Cambridge: MIT Press.

Habermas, J. (1998). Between Facts and Norms: Contributions to a Discourse Theory of Law and Democracy, tradução de William Rehg, Cambridge: MIT Press. [Facticidade e Validade: Contribuições Para Uma Teoria Discursiva do Direito e da Democracia. Tradução de Rúrion Melo e Felipe Gonçalves. $1^{a}$ edição. Editora Unesp, 2020.]

Habermas, J. (1998). The Inclusion of the Other: Studies in Political Theory. Ed. Ciaran Cronin e Pablo de Greiff. Cambridge: MIT Press [A inclusão do outro: estudos de teoria política. Tradução de Denilson Luis Werle. $1^{a}$ Edição. Editora Unesp, 2018]

Habermas, J. (2001). "The Postnational Constellation and the Future of Democracy" em The Postnational Constellation: Political Essays, p. 98-103. Cambridge: Polity Press. [A constelação pós-nacional: ensaios políticos. Tradução de Márcio Seligmann-Silva. São Paulo: Littera Mundi, 2001]

Habermas, J. (2006a). “Does Europe Need a Constitution?” in Time of Transitions, ed. e trad. Ciaran Cronin and Max Pensky, p. 102-106. Cambridge: Polity Press. [A inclusão do outro: estudos de teoria política. Tradução de Denilson Luis Werle.

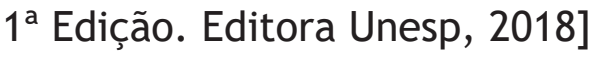

Habermas, J. (2006b). "Does the Constitutionalization of International Law Still Have a Chance?" em The Divided West, p. 142-143. Cambridge: Polity Press. [0 Ocidente dividido. Tradução de Bianca Tavolari. 1ª Edição. Editora Unesp, 2016].

Habermas, J. (2006b). "Is the Development of a European Identity Necessary, and Is It Possible?” em The Divided West. Cambridge: Polity Press [O Ocidente dividido. Tradução de Bianca Tavolari. $1^{\text {a }}$ Edição. Editora Unesp, 2016].

Hutchings, K. (2007). Whose history? Whose justice? Theory, Culture \& Society, 24(4), 59-63. DOI: https://doi.org/10.1177/0263276407080094

Mccarthy, T. (2009). Race, empire, and the idea of human development. Cambridge: Cambridge University Press.

Randeria, S. (2007). De-politicization of Democracy and Judicialization of Politics. Theory, Culture \& Society, 24(4), 38-44. DOI: https://doi. org/10.1177/0263276407080398.

Salvatore, A. (2007). The exit from a Westphalian framing of political space and the emergence of a transnational Islamic public. Theory, Culture \& Society, 24(4), 45-52. DOI: https://doi.org/10.1177/0263276407080092.

Scheuerman, W. (2008). Global Governance without Global Government? Habermas on Postnational Democracy. Political Theory, 36(1), 133-151. DOI: https://doi. org/10.1177/0090591707310100. 
Ugarteche, O. (2007). Transnationalizing the public sphere: Acritique of Fraser. Theory, Culture\& Society, 24(4), 65-69. DOI: https: / / doi.org/10.1177/0263276407080095.

Recebido em: 31.03.2021

Aceito em: 19.07.2021

Isenção de responsabilidade SAGE Publication ${ }^{1}$

1 "Embora todos os esforços tenham sido feitos para garantir que o conteúdo desta publicação seja factualmente correto, nem os autores, nem a editora aceitam, e por meio deste expressamente excluem, em toda a extensão permitida pela legislação aplicável, toda e qualquer responsabilidade decorrente dos conteúdos publicados neste artigo, incluindo, sem limitação, quaisquer erros, omissões, imprecisões na tradução original ou posterior, ou por quaisquer consequências daí decorrentes. Nenhuma disposição do presente aviso exclui a responsabilidade que não possa ser excluída por lei. As informações do produto aprovado devem ser revisadas antes de prescrever qualquer medicamento em questão".

"While every effort has been made to ensure that the contents of this publication are factually correct, neither the authors nor the publisher accepts, and they hereby expressly exclude to the fullest extent permissible under applicable law, any and all liability arising from the contents published in this Article, including, without limitation, from any errors, omissions, inaccuracies in original or following translation, or for any consequences arising therefrom. Nothing in this notice shall exclude liability which may not be excluded by law. Approved product information should be reviewed before prescribing any subject medications" (N. T). 